\title{
miR-491-3p suppresses the growth and invasion of osteosarcoma cells by targeting TSPAN1
}

\author{
JUNHU DUAN $^{1 *}$, JIANGTAO LIU $^{1 *}$, YIN LIU $^{2}$, BIN HUANG $^{1}$ and LONGHUA RAO ${ }^{2}$ \\ Departments of ${ }^{1}$ Orthopedics and ${ }^{2}$ Nephrology, Xiangyang No. 1 People's Hospital, \\ Hubei University of Medicine, Xiangyang, Hubei 441000, P.R. China
}

Received February 16, 2016; Accepted February 21, 2017

DOI: $10.3892 / \mathrm{mmr} .2017 .7256$

\begin{abstract}
Osteosarcoma (OS) is the most common primary bone cancer, and frequently leads to mortality as a result of rapid proliferation and metastasis. Recent data highlight the important role of microRNAs (miRs) in human cancers, including OS, providing a novel method for its diagnosis and treatment. In the present study, it was identified that miR-491-3p expression was frequently decreased in OS tissues and OS cell lines. Restored miR-491-3p expression suppressed the growth and invasion of OS cells. Bioinformatics and experimental analysis indicated that tetraspanin 1 (TSPAN1) is a direct target of miR-491-3p in OS. The TSPAN1 mRNA level was inversely associated with the miR-491-3p level in OS tissues and cell lines. Consistent with this, TSPAN1 knockdown inhibited the growth and invasion of OS cells. In conclusion, these data provide compelling evidence that miR-491-3p functions as a tumor suppressor in OS to attenuate the potential of growth and invasion by targeting TSPAN1.
\end{abstract}

\section{Introduction}

Osteosarcoma (OS) is the most common malignant primary bone tumor in children and represents $\sim 6 \%$ of all childhood cancers (1). OS is locally destructive and also has a high metastatic potential, primarily leading to secondary cancer of the lung (1). During the past decades, great efforts have been exerted to interpret the underlying mechanisms for OS progression and the rapid development of treatment strategies have been achieved; however, the prognosis of OS remains poor $(2,3)$. Therefore, the identification of novel diagnostic biomarkers and therapeutic targets is required for patients with OS.

Correspondence to: Dr Longhua Rao, Department of Nephrology, Xiangyang No. 1 People's Hospital, Hubei University of Medicine, Xiangyang, Hubei 441000, P.R. China

E-mail: longhuaraoxy@126.com

${ }^{*}$ Contributed equally

Key words: osteosarcoma, miR-491-3p, tetraspanin 1, tumor growth, invasion
MicroRNAs (miRNAs/miRs) are a class of small non-coding RNAs, which serve a crucial role in regulating gene expression by targeting mRNAs for translational repression or degradation (4). Abnormalities of miRNAs have been implicated in many physiological and pathophysiological processes, including development, proliferation, differentiation, apoptosis, migration and invasion, especially in carcinogenesis $(4,5)$. Overexpression of oncogenic miRNAs or low-expression of tumor suppressor miRNAs are crucial in cancer development and progression $(5,6)$. In addition, certain recent reports have suggested that there is involvement of certain dysregulated miRNAs in OS, such as miR-135b (3), miR-205 (7) and miR-155 (8), yet the roles of these miRNAs in OS require further elucidation. Previously, miR-491-3p was reported to function as a tumor suppressor, and was identified as being downregulated in retinoblastoma cells under hypoxia, which is critical for retinoblastoma progression (9). Furthermore, miR-491-3p expression was downregulated in glioblastoma multiformed samples. Ectopic overexpression of miR-491-3p inhibited glioma cell invasion and proliferation and impaired the propagation of glioma stem cells (10). A report from Zheng et al (11) indicated that reduced miR-491-3p confers chemo-resistance in tongue cancer cells via enhanced mTORC2 activation. In the present study, the expression of miR-491-3p in OS tissues and cell lines was investigated, and a series of cellular and molecular function experiments were performed, to investigate the role and mechanisms of miR-491-3p in OS cell growth and invasion.

\section{Materials and methods}

Specimens. A total of 14 samples of osteosarcoma tissues were collected between March 2012 and September 2014 at Xiangyang No. 1 People's Hospital (Xiangyang, China). A total of 9 male and 5 female patients were involved in the study, at an average age of 21-32 years. The matched normal tissues were obtained $5 \mathrm{~cm}$ distance away from the tumor margin, which were further confirmed by pathologists. All patients did not undergo any therapy prior to recruitment for this research. Each of the patients provided written informed consent and the present study was approved by the ethics committee of Xiangyang No. 1 People's Hospital.

A total of 20 female $\mathrm{BALB} / \mathrm{C}$ athymic nude mice (4-6-weeks-old) were purchased from Hubei Research Center of 
Laboratory Animals (Wuhan, China). All mice were housed and maintained under specific pathogen-free conditions at $27^{\circ} \mathrm{C}$ with $12 \mathrm{~h}$ light/dark cycle, with free access to food and water, and all experiments were approved by the Experimental Animal Ethics Committee of Hubei University of Medicine (Shiyan, China) and performed in accordance with institutional guidelines.

Cell culture and transfection. The human OS cell lines (HOS, Saos-2, U2OS and MG-63) and normal osteoblast cells (NHOst) were obtained from the American Type Culture Collection (Manassas, VA, USA). The cells were maintained in Dulbecco's modified Eagle's medium (Gibco; Thermo Fisher Scientific, Inc., Waltham, MA, USA), supplemented with $10 \%$ fetal bovine serum (FBS; GE Healthcare Life Sciences, Chalfont, UK) and streptomycin $(100 \mu \mathrm{g} / \mathrm{ml})$, penicillin $(100 \mathrm{U} / \mathrm{ml})$. Cultures were incubated in a humidified atmosphere containing $5 \% \mathrm{CO}_{2}$ at $37^{\circ} \mathrm{C}$. For miR-491-3p overexpression and TSPAN1 knockdown, cells were transfected with miR-491-3p overexpressing vector HIVH1-miR-491-3p and pSUPER vector containing TSPAN1 specific short hairpin (sh)RNAs, respectively (Guangzhou Fulengen Co., Ltd., Guangzhou, China), using Lipofectamine 2000 (Invitrogen; Thermo Fisher Scientific, Inc.) in accordance with the manufacturer's protocol.

Reverse transcription-quantitative polymerase chain reaction (RT-qPCR) for mature miRNAs and mRNA. miRNAs from cultured cells and tissues were isolated and purified using the miRNA isolation system (Exiqon A/S, Vedbaek, Denmark). cDNA was generated using the miScript II RT kit (Qiagen $\mathrm{GmbH}$, Hilden, Germany), and qPCR was performed using the miScript SYBR Green PCR kit (Qiagen $\mathrm{GmbH}$ ) following the manufacturer's instructions. The miRNA sequence-specific RT-qPCR primers and the endogenous control RNU6 primers were purchased from Qiagen $\mathrm{GmbH}$. The relative quantitative expression was calculated by normalizing the results with RNU6. The RT-qPCR analysis was conducted using the 7500 Real-Time PCR system (Applied Biosystems; Thermo Fisher Scientific, Inc.). The total RNA was extracted according to the TRIzol reagent (Thermo Fisher Scientific, Inc.), and cDNAs from the mRNAs were synthesized with the first-strand synthesis system (Thermo Fisher Scientific, Inc.). qPCR was carried out according to standard protocols using an ABI 7500 with SYBR-Green detection (Applied Biosystems; Thermo Fisher Scientific, Inc.). The primers used were as follows: GAPDH forward primer, 5'-ATTCCATGGCACCGT CAAGGCTGA-3'; GAPDH reverse primer, 5'-TTCTCCATG GTGGTGAAGACGCCA-3'; tetraspanin 1 (TSPAN1) forward primer, 5'- CATGCAGTTTGTCAACGTGGG-3'; TSPAN1 reverse primer, 5'- CACTTGCTCTCAGTCTTAGCAC-3'. The thermocycling conditions for the $\mathrm{qPCR}$ reactions were as follows: 1 cycle at $95^{\circ} \mathrm{C}$ for $5 \mathrm{~min} ; 40$ cycles at $95^{\circ} \mathrm{C}$ for $45 \mathrm{sec}$, $61^{\circ} \mathrm{C}$ for $30 \mathrm{sec}$ and $72^{\circ} \mathrm{C}$ for $30 \mathrm{sec}$ followed by 1 cycle at $72^{\circ} \mathrm{C}$ for $10 \mathrm{~min}$. The gene expression threshold cycle values of miRNAs or mRNAs were calculated by normalization with internal control RNU6 or GAPDH, and relative quantization values were calculated using the $2^{-\Delta \Delta \mathrm{Cq}}$ method (12).

Cell proliferation assay. Cell proliferation was monitored using the MTS assay using the CellTiter96 ${ }^{\circledR}$ AQueous One Solution Cell Proliferation Assay kit (Promega Corporation,
Madison, WI, USA) according to the manufacturer's instructions. Cells were seeded into 96-well plates at 3,000 cells/well (200 $\mu \mathrm{l} /$ well). The cell proliferation assay was performed on days $0,1,2,3,4$ and 5 by incubation with MTS (20 $\mu \mathrm{l} /$ well). Following $2 \mathrm{~h}$ further incubation, the absorbance at $490 \mathrm{~nm}$ was recorded for each well on the BioTek Synergy 2 (BioTek Instruments, Inc., Winooski, VT, USA). The absorbance reflected the cell number.

Transwell assay. The migratory ability of cells was evaluated using the Cell Invasion Assay kit (BD Biosciences, Franklin Lakes, NJ, USA) following the manufacturer's protocol. A total of $3 \times 10^{4}$ cells in $300 \mu 1$ serum-free medium were added to the upper chamber precoated with ECMatrix ${ }^{\text {TM }}$ gel. A total of $0.5 \mathrm{ml}$ medium with $10 \%$ FBS was added to the lower chamber as a chemoattractant. Subsequently, cells were incubated at $37^{\circ} \mathrm{C}$ for $24 \mathrm{~h}$. Non-invading cells were removed with cotton swabs. Cells that migrated to the bottom of the membrane were fixed with pre-cold methanol and stained with $2 \%$ Giemsa solution. Stained cells were visualized under a light microscope (LEICA DM6000; Leica Microsystems $\mathrm{GmbH}$, Wetzlar, Germany). To minimize the bias, at least three randomly selected fields with 100x magnification were counted, and the average number was taken.

Western blot analysis. Total proteins were extracted from corresponding cells using radioimmunoprecipitation assay buffer (Thermo Fisher Scientific, Inc.) in the presence of a Protease Inhibitor cocktail (Thermo Fisher Scientific, Inc.). The protein concentration of the lysates was measured using a Bicinchoninic Acid Protein Assay kit (Thermo Fisher Scientific, Inc.). $50 \mu \mathrm{g}$ protein was resolved and mixed with 5X Lane Marker Reducing Sample Buffer, electrophoresed on a 10\% SDS-PAGE gel and transferred onto an Immobilon-P Transfer membrane (EMD Millipore, Billerica, MA, USA). The membranes were blocked with $5 \%$ non-fat milk in Tris-buffered saline at $25^{\circ} \mathrm{C}$ for $2 \mathrm{~h}$. Then, the membranes incubated with primary antibodies at $4^{\circ} \mathrm{C}$ overnight, followed by secondary antibody at $25^{\circ} \mathrm{C}$ for $2 \mathrm{~h}$. The signal was detected using an ECL detection system (34077; SuperSignal West Pico Chemiluminescent Substrate, Merck KGaA, Darmstadt, Germany). The TSPAN1 antibody (NBP2-33867; 1:1,000) was obtained from Novus Biologicals, LLC (Littleton, CO, USA) and the GAPDH antibody (5147; 1:1,000) was from Cell Signaling Technology, Inc. (Danvers, MA, USA). The horseradish peroxidase-conjugated secondary antibody (31460; 1:20,000) was from Thermo Fisher Scientific, Inc.

Xenograft model in nude mice. Xenograft tumors were generated by subcutaneous injection of U2OS/Control, U2OS/miR-491-3p, U2OS/sh-Control and U2OS/sh-TSPAN1 cell lines $\left(2 \times 10^{6}\right.$ cells) into the flank of $4-6$ week-old BALB/c mice. Tumor formation was examined every 3 days for the whole duration of the experiment. The mice were sacrificed with $120 \mu 1$ 10\% hydral (Sinopharm Chemical Reagent Co., Ltd, Shanghai, China) after 27 days and the tumors were harvested and weighed.

Luciferase reporter assay. The 3'-untranslated region (UTR) of the TSPAN1 gene was synthesized and cloned into the 
A

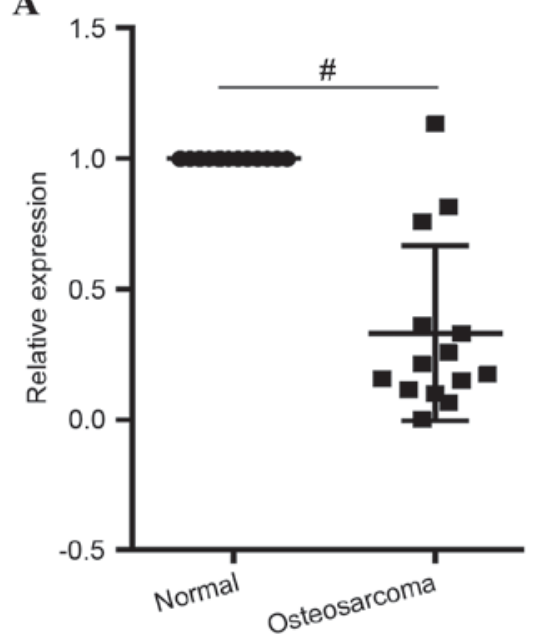

B

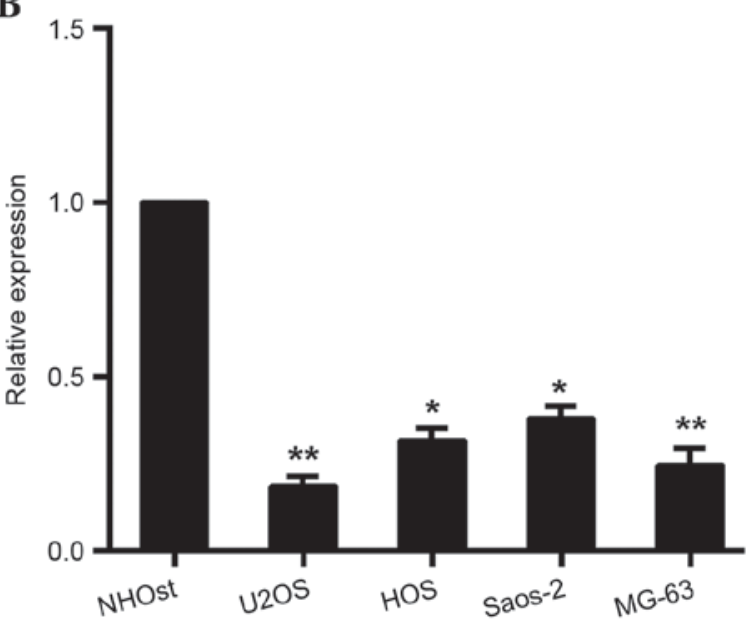

Figure 1. miR-491-3p expression in OS tissues and cell lines. (A) miR-491-3p expression in 14 pairs of OS tissues and adjacent normal bone tissues was determined using RT-qPCR. Data was presented as the relative ratio change of OS tissues relative to normal bone tissues. " $\mathrm{P}<0.0001$ vs. normal tissues. (B) Using RT-qPCR analysis, the expression levels of miR-491-3p in OS cell lines (U2OS, HOS, Saos-2 and MG-63) were determined. ${ }^{*} \mathrm{P}<0.01,{ }^{* *} \mathrm{P}<0.0001$ vs. NHOst cells. OS, osteosarcoma; miR, microRNA; RT-qPCR, reverse transcription-quantitative polymerase chain reaction; NHOst, normal osteoblast cells.
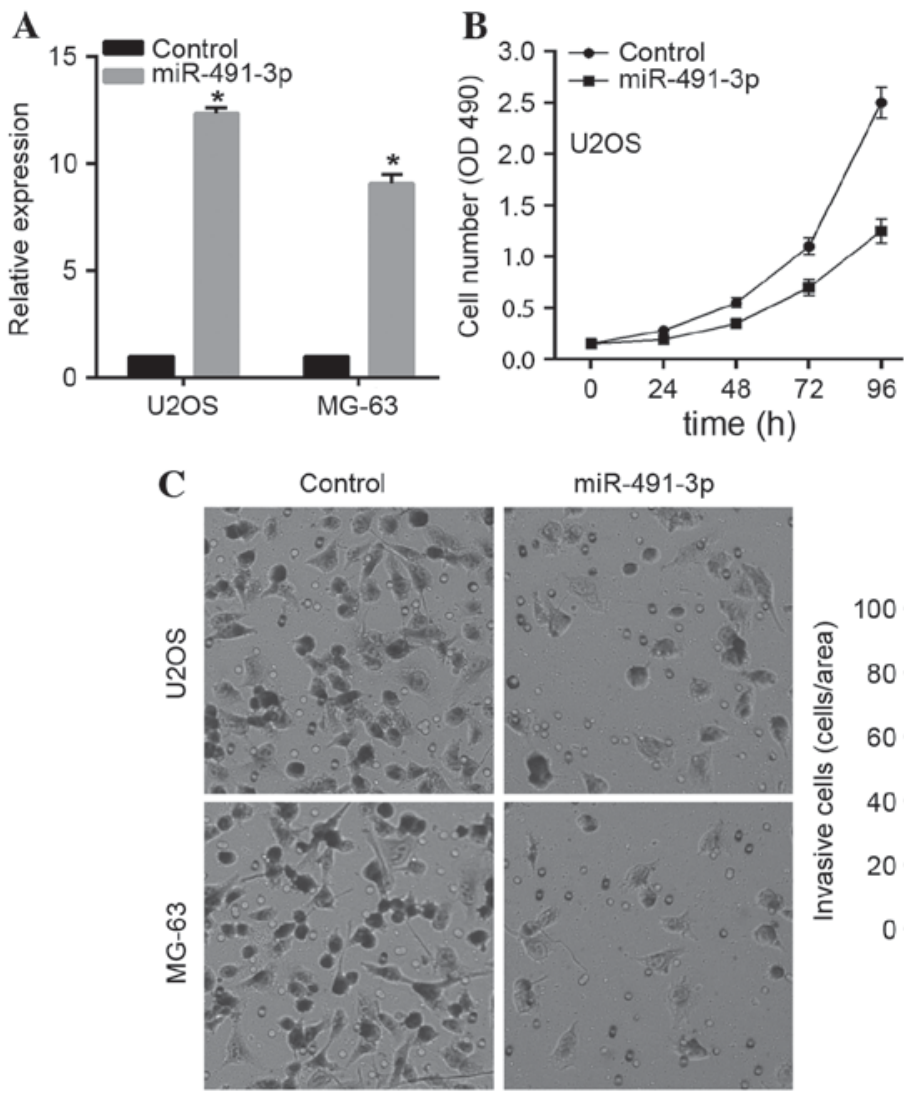

miR-491-3p

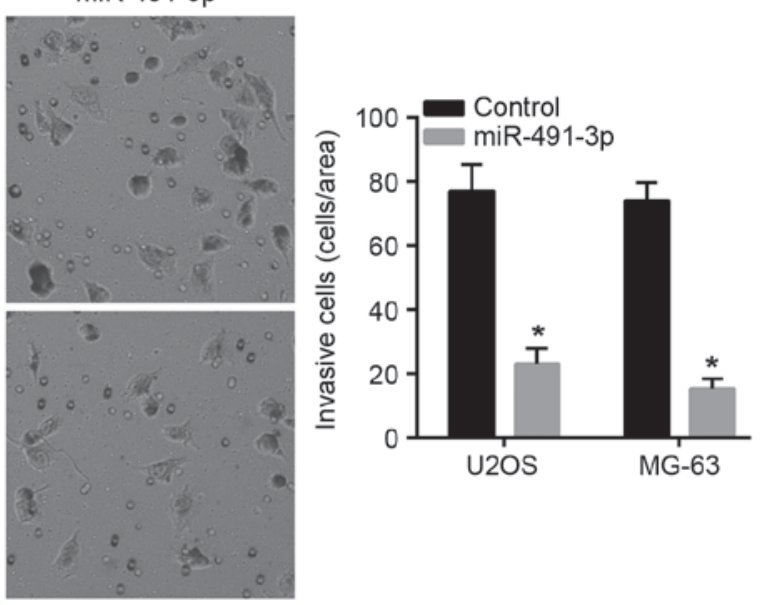

Figure 2. Effects of miR-491-3p on proliferation and invasion in OS cells. (A) The relative miR-491-3p expression level in U2OS and MG-63 cell lines using the reverse transcription-quantitative polymerase chain reaction following transfection with miR-491-3p overexpressing the vector or control. (B) The proliferation patterns of U2OS and MG-63 cells transfected with miR-491-3p overexpressing vector or control were determined using MTS assays. (C) The invasion potential of U2OS and MG-63 cell lines overexpressing miR-491-3p was examined by Transwell invasion assays. "P<0.001 vs. control. miR, microRNA; OS, osteosarcoma.

pMir-Report-vector immediately downstream of the firefly luciferase gene (wild type, pMir-WT) (Promega Corporation). Mutations in the 3'-UTR of TSPAN1 gene with miR-491-3p target sites deleted (mutant type, pMir-Mut) were generated with the QuickChange Site-Directed Mutagenesis kit
(Stratagene; Agilent Technologies, Inc., Santa Clara, CA, USA). Cells were seeded in 96-well plates (6000 cells/well) and co-transfected with the pMir-Report luciferase vector, pRL-TK Renilla luciferase vector and miR-135b inhibitor or control. A total of $48 \mathrm{~h}$ later, the luciferase activities were 

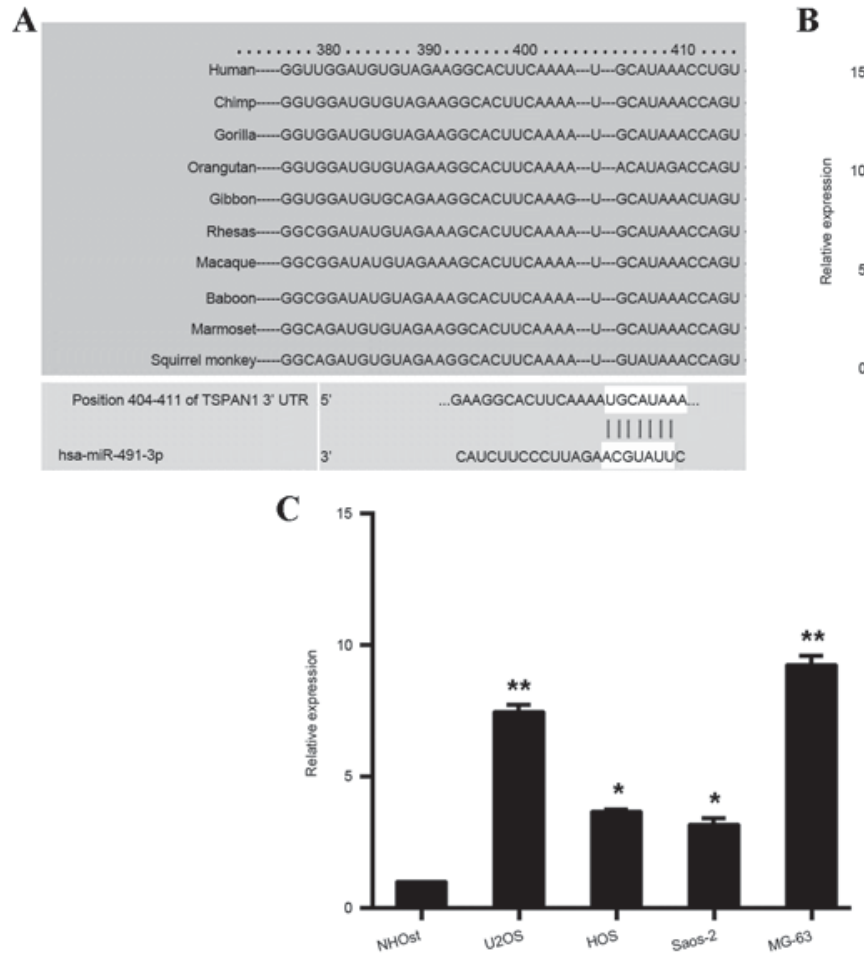

D

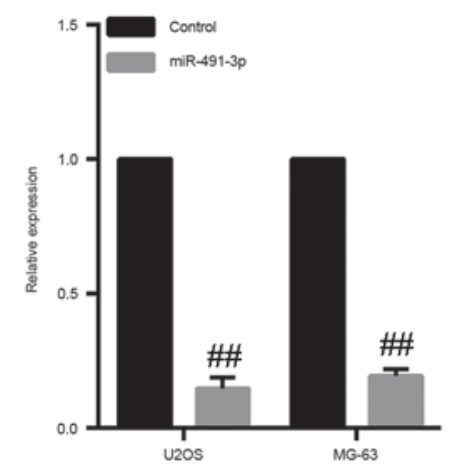

$\mathbf{B}$

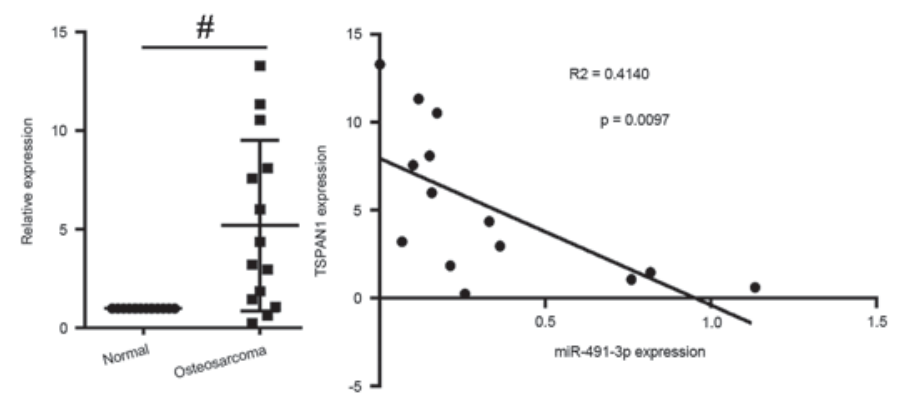

Figure 3. miR-491-3p targets TSPAN1 in OS cells. (A) Schematic representation of the putative binding sites of miR-491-3p in 3'-UTR of TSPAN1, which is broadly conserved among vertebrates. (B) The relative TSPAN1 expression level in OS tissues was examined by RT-qPCR. TSPAN1 level was negatively correlated with miR-491-3p level in OS tissues. ${ }^{*} \mathrm{P}<0.0001$ vs. normal. (C) TSPAN1 expression in mRNA and protein levels in OS cell lines were determined by RT-qPCR and western blotting respectively. ${ }^{*} \mathrm{P}<0.01,{ }^{* * *} \mathrm{P}<0.0001$ vs. NHOst. (D) TSPAN1 expression change in mRNA and protein levels in OS cells following miR-491-3p overexpression were detected using RT-qPCR and western blotting. ${ }^{\# \#} \mathrm{P}<0.0001$ vs. control. (E) Luciferase reporter assay in U2OS and MG-63 cells cotransfected with miR-491-3p expressing vector or control, a luciferase reporter containing wild-type TSPAN1 3'-UTR or a mutant version, and a Renilla luciferase reporter for normalization. The mean of the results from cells transfected with expression control vector and pMir-con was set as $100 .{ }^{* * *} \mathrm{P}<0.001$ vs. pMir-Wt. miR, microRNA; OS, osteosarcoma; RT-qPCR, reverse transcription-quantitative polymerase chain reaction; NHOst, normal osteoblast cells; con, control; Wt, wild-type; mut, mutated.

determined using a Dual-Luciferase Reporter Assay system (Promega Corporation) where the Renilla luciferase activity was used as internal control and the firefly luciferase activity was calculated as the mean \pm standard deviation following normalization to Renilla luciferase activity.

Immunohistochemistry. Immunohistochemistry was used to detect TSPAN1 expression in the tissue of the xenograft mouse model tumors. The tissues were fixed as Swiss-roll in $10 \%$ formalin overnight. The $4 \mu \mathrm{m}$-thick sections were cut from formalin-fixed paraffin-embedded tissue blocks and subsequently deparaffinized in xylene and rehydrated in successive washes of ethanol. The tissue slides were then treated with $3 \%$ hydrogen peroxide in methanol for $15 \mathrm{~min}$ to quench endogenous peroxidase activity, followed by antigen retrieval performed via incubation at $98-100^{\circ} \mathrm{C}$ for $10 \mathrm{~min}$ in $0.01 \mathrm{M}$ sodium citrate buffer ( $\mathrm{pH}$ 6.0), heated using a microwave oven. Following pre-incubation for $1 \mathrm{~h}$ in $10 \%$ goat serum (Gibco; Thermo Fisher Scientific, Inc.), the specimens were incubated with TSPAN1 antibody (NBP2-33867; 1:50) obtained from Novus Biologicals, for $1 \mathrm{~h}$ at room temperature. The tissue slides were treated with a non-biotin horseradish peroxidase detection system according to the manufacturer's instruction (Dako; Agilent Technologies, Inc.). There were two different pathologists who evaluated the immunohistological samples.

Statistical analysis. Data were expressed as the mean \pm standard deviation of at least three independent experiments. Statistical 
A

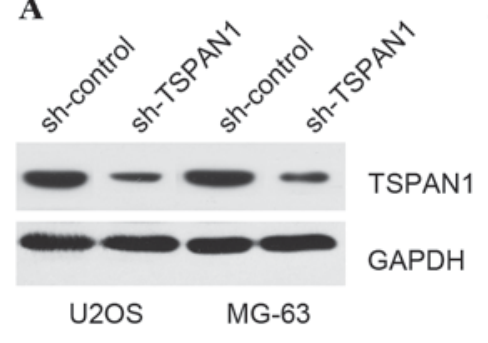

C

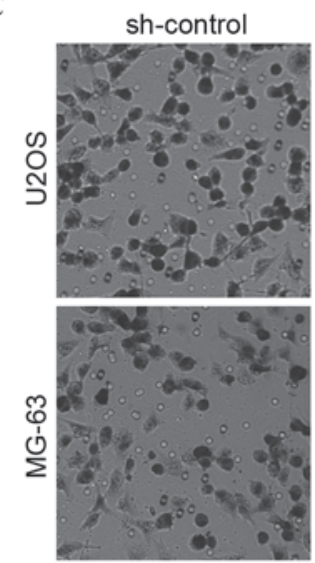

B

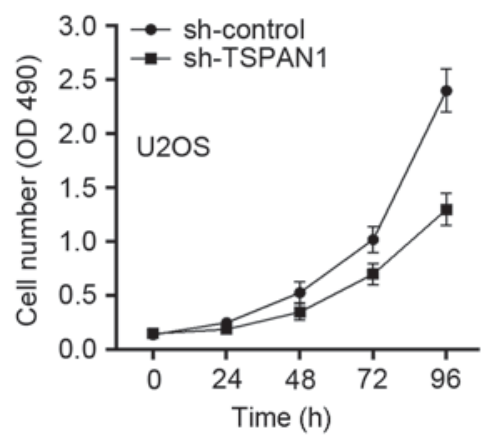

Sh-TSPAN1

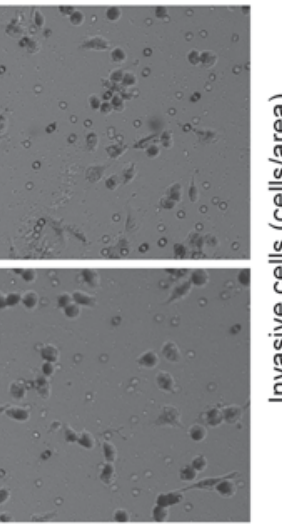

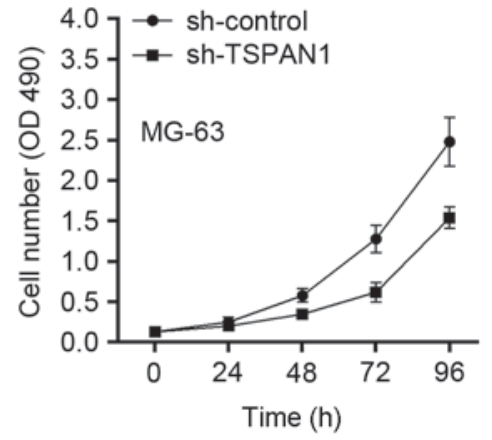

Figure 4. TSPAN1 knockdown inhibits OS cells proliferation and invasion. (A) Using sh-RNAs, TSPAN1 was downregulated in OS cell lines detected by western blot analysis. (B) TSPAN1 knockdown inhibited OS cells proliferation determined with MTS proliferation assay. (C) TSPAN1 knockdown inhibited invasive potential of OS cells examined by Transwell assay. ${ }^{*} \mathrm{P}<0.001$ vs. control. OS, osteosarcoma; shRNA, short hairpin RNA.

analysis was carried out using SPSS software (version 18.0; SPSS Inc., Chicago, IL, USA) and Student's t-tests were used to calculate the $\mathrm{P}$-value. $\mathrm{P}<0.05$ was considered to indicate a statistically significant difference.

\section{Results}

miR-491-3p is downregulated in OS tissues and cell lines. To investigate the expression and significance of miR-491-3p in OS carcinogenesis, miR-491-3p expression was initially detected in 14 pairs of OS tissues and the matched normal tissues using RT-qPCR analysis. miR-491-3p was significantly downregulated in 11 of the 14 OS tissues when compared with the matched normal control (Fig. 1A). Furthermore, the miR-491-3p level in OS cell lines was evaluated. The results indicated that the miR-491-3p level was reduced in OS cell lines U2OS, HOS, Saos-2 and MG-63, when compared with the normal human osteoblast cell line, NHOst (Fig. 1B). These results suggested that $\mathrm{miR}-491-3 \mathrm{p}$ is frequently downregulated in OS.

miR-491-3p suppresses OS cells proliferation and invasion. The downregulation of miR-491-3p in OS tissues and cell lines prompted the investigation of its potential biological role in OS progression. In the present study, miR-491-3p was overexpressed stably in OS cell lines U2OS and MG-63 by transfection with the miR-491-3p overexpressing vector, HIVH1-miR-491-3p (Fig. 2A). In vitro cell proliferation was measured using an MTS assay. Overexpressed miR-491-3p significantly inhibited the proliferation potential of U2OS and MG-63 cell lines, when compared with the control group (Fig. 2B). In addition, results from the Transwell assay demonstrated that miR-491-3p markedly attenuated the invasive potential of OS cells, when compared with its control (Fig. 2C). These data implied that miR-491-3p downregulation was involved in OS progression.

miR-491-3p targets TSPAN1 expression in OS cells. miRNAs primarily exert their roles by suppressing target genes post-transcriptionally. In order to identify novel targets of miR-491-3p in OS cells, the potential targets were predicted using database-TargetScan (www.targetscan. org) and TSPAN1, with a critically conserved binding site, was selected for further validation (Fig. 3A). The TSPAN1 mRNA level in OS tissues was examined using RT-qPCR. The results indicated that TSPAN1 mRNA level was upregulated in OS tissues, when compared with the normal control, which was negatively correlated with miR-491-3p level (Fig. 3B). Similarly, TSPAN1 mRNA and protein levels were upregulated in OS cell lines HOS, Saos-2, U2OS and MG-63, when compared to NHOst (Fig. 3C). To investigate whether miR-491-3p may target TSPAN1 in OS cells, TSPAN1 mRNA and protein expression was reduced in in U2OS and MG-63 cells following miR-491-3p overexpression (Fig. 3D). To validate the direct interaction between miR-491-3p and TSPAN1 in OS cells, the 3'-UTR of TSPAN1 with its putative binding site was cloned into the luciferase reporter vector set as wild-type version (pMir-Wt), and 3'-UTR with a deletion 

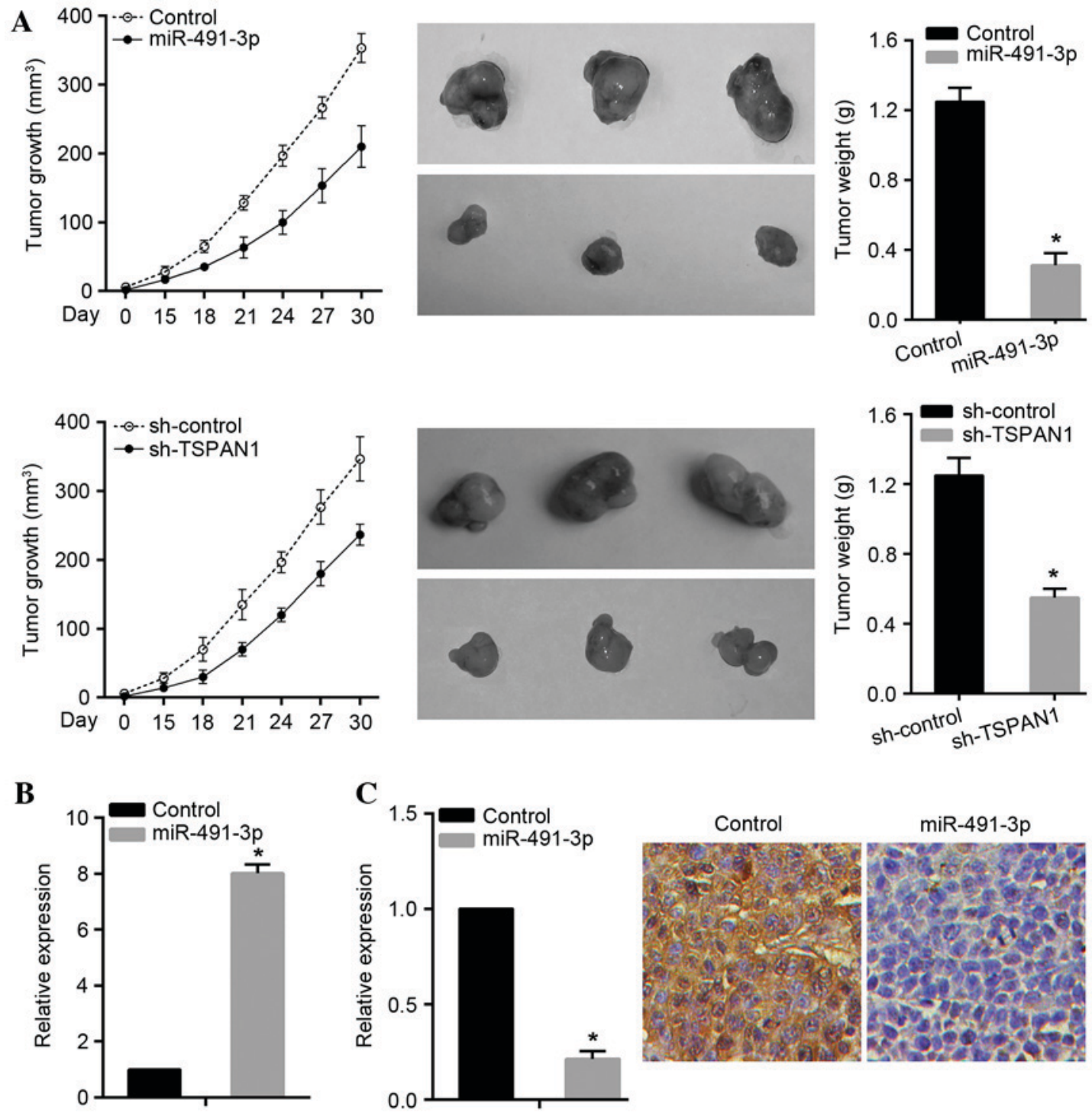

Figure 5. miR-491-3p overexpression and TSPAN1 knockdown suppresses OS growth in vivo. (A) miR-491-3p overexpression or TSPAN1 knockdown in U2OS cells and their related control cells were subcutaneously injected in the dorsal flank of nude mice. A total of 30 days later, U2OS cells with miR-491-3p overexpression or TSPAN1 knockdown had smaller tumors than controls. (Left) The growth curves tumor volume. (Middle) Representative images of tumors. (Right) The average tumor weight. (B) Expression of miR-491-3p in the xenograft tumors was detected by RT-qPCR. (C) Expression of TSPAN1 mRNA and protein in the xenograft tumor was detected by RT-qPCR and immunohistochemistry, respectively. ${ }^{\text {P }}<0.001$ vs. control. miR, microRNA; OS, osteosarcoma; miR, microRNA; RT-qPCR, reverse transcription-quantitative polymerase chain reaction.

of $7 \mathrm{bp}$ in the seed region was constructed as mutant version (pMir-Mut). As expected, significant repression of luciferase activities was observed in U2OS and MG-63 cells following co-transfection with the pMir-Wt vector and miR-491-3p overexpressing vector, when compared to control groups (Fig. 3E). These results indicated that miR-491-3p may direct target TSPAN1 in OS cells.

TSPAN1 knockdown inhibits OS cells proliferation and invasion. Given that, as a target of miR-491-3p, TSPAN1 expression was upregulated in OS, it was necessary to observe the role of TSPAN1 in OS progression. TSPAN1 specific shRNAs was used to knockdown TSPAN1 expression in U2OS and MG-63 cell lines (Fig. 4A). TSPAN1 knockdown markedly inhibited the proliferation (Fig. 4B). Furthermore, TSPAN1 knockdown significantly reduced the invasive potential of U2OS and MG-63 cell lines (Fig. 4C).
miR-491-3p overexpression and TSPAN1 knockdown inhibit OS growth in vivo. To further investigate whether miR-491-3p and TSPAN1 affects OS growth in vivo, a xenograft tumors model was established either by subcutaneous injection of U2OS/Control, U2OS/miR-491-3p, U2OS/sh-Control and U2OS/sh-TSPAN1 cell lines into the dorsal flank of nude mice. It was demonstrated that tumors from U2OS cells with miR-491-3p overexpression or TSPAN1 knockdown grew at a slower rate and had smaller volumes compared with the control groups (Fig. 5A). Then, to observe whether the regulation of TSPAN1 by miR-491-3p also exists in vivo, the miR-491-3p and TSPAN1 levels were determined by RT-qPCR and immunohistochemistry, respectively, in OS xenografts from miR-491-3p overexpression and control groups. As demonstrated in the figure, compared to the control group, the miR-491-3p level in the miR-491-3p overexpression group was increased (Fig. 5B), whereas, the TSPAN1 expression in 
mRNA and protein levels were significantly downregulated (Fig. 5C). These results suggested that miR-491-3p suppresses OS growth and the TSPAN1 expression in vivo.

\section{Discussion}

Rapid growth and metastasis are the major causes leading to the majority of cancer-associated mortalities, therefore it is pivotal to explore and develop strategies based on targeting proliferation and metastasis in anticancer treatment. The involvement of miRNAs in carcinogenesis and progression has been well documented in all human cancer types via regulating the expression or function of many oncogenes and tumor suppressors (6). The role of miR-491-3p in cancer has been reported only in a few studies (9-11). In the present study, important evidence was provided in support of miR-491-3p functioning as a tumor suppressor in OS in vitro and in vivo. TSPAN1 was identified to be a novel target of miR-491-3p in OS cells. In agreement, TSPAN1 knockdown also inhibits OS growth in vitro and in vivo.

TSPAN1 is a member of the tetraspanin family, which is involved in various fundamental biochemical processes, including cell development, differentiation, proliferation, adhesion, migration and intracellular signal transduction $(13,14)$. TSPAN1 is a recently discovered tumor-associated gene and has been reported to regulate cancer progression in several human cancer types. Types of cancer in which TSPAN1 was observed to be overexpressed include those of the cervix (15), esophagus (16), colon (17), liver (18), ovary (19), skin (20) and stomach (21). In colorectal cancer, TSPAN1 promotes cells growth and invasion. High TSPAN1 expression levels in colorectal cancer tissues predicted poor overall survival, appearing to be an independent prognostic factor (17). In skin carcinoma, knockdown of TSPAN1 inhibited the proliferation and infiltration of human skin squamous carcinoma cells (20). The TSPAN1 expression level is significantly increased in gastric cancer tissues, when compared with non-cancerous tissues. TSPAN1 knockdown markedly blocks gastric cancer cells proliferation, cell cycle progression and invasive activity (21). Consistent with these results, TSPAN1 was identified in the present study to be overexpressed in OS cells. TSPAN1 knockdown suppressed OS cell growth in vitro and in vivo, and also inhibited the invasive potential. With regards to the mechanisms underlying TSPAN1 upregulation in cancer cells, there are only a few reports. In colorectal cancer, miR-638 downregulation led to TSPAN1 overexpression (17). In the present study, the TSPAN1 expression level was negatively correlated with miR-491-3p level in OS. Overexpressed miR-491-3p significantly reduced TSPAN1 expression in mRNA and protein levels in OS cells. The experimental results indicated that TSPAN1 was a novel direct target of miR-491-3p. For further study, the authors of the present study intend to investigate the mechanisms of miR-491-3p downregulation, as well as understand how TSPAN1 exerts its oncogenic role in OS.

In conclusion, miR-491-3p is frequently downregulated in OS. miR-491-3p suppressed OS cell growth and invasion. TSPAN1 was identified to be a novel target of miR-491-3p. Overexpressed TSPAN1 conferred OS cell growth and invasion. These results indicated that dysregulation of miR-491-3p/TSPAN1 confers OS progression.

\section{References}

1. He H, Ni J and Huang J: Molecular mechanisms of chemoresistance in osteosarcoma (Review). Oncol Lett 7: 1352-1362, 2014.

2. Janeway KA, Barkauskas DA, Krailo MD, Meyers PA, Schwartz CL, Ebb DH, Seibel NL, Grier HE, Gorlick R and Marina N: Outcome for adolescent and young adult patients with osteosarcoma: A report from the Children's Oncology Group. Cancer 118: 4597-4605, 2012.

3. Pei H, Jin Z, Chen S, Sun X, Yu J and Guo W: MiR-135b promotes proliferation and invasion of osteosarcoma cells via targeting FOXO1. Mol Cell Biochem 400: 245-252, 2015.

4. Ambros V: microRNAs: Tiny regulators with great potential. Cell 107: 823-826, 2001.

5. Calin GA and Croce CM: MicroRNA signatures in human cancers. Nat Rev Cancer 6: 857-866, 2006.

6. Croce CM: Causes and consequences of microRNA dysregulation in cancer. Nat Rev Genet 10: 704-714, 2009.

7. Yang G, Zhang P, Lv A, Liu Y and Wang G: MiR-205 functions as a tumor suppressor via targeting TGF- $\alpha$ in osteosarcoma. Exp Mol Pathol 100: 160-166, 2015.

8. Sun X, Geng X, Zhang J, Zhao H and Liu Y: miR-155 promotes the growth of osteosarcoma in a HBP1-dependent mechanism. Mol Cell Biochem 403: 139-147, 2015.

9. Xu X, Jia R, Zhou Y, Song X, Wang J, Qian G, Ge S and Fan X: Microarray-based analysis: Identification of hypoxia-regulated microRNAs in retinoblastoma cells. Int J Oncol 38: 1385-1393, 2011.

10. Li X, Liu Y, Granberg KJ, Wang Q, Moore LM, Ji P, Gumin J, Sulman EP, Calin GA, Haapasalo H, et al: Two mature products of MIR-491 coordinate to suppress key cancer hallmarks in glioblastoma. Oncogene 34: 1619-1628, 2015.

11. Zheng G, Jia X, Peng C, Deng Y, Yin J, Zhang Z, Li N, Deng M, Liu X, Liu H, et al: The miR-491-3p/mTORC2/FOXO1 regulatory loop modulates chemo-sensitivity in human tongue cancer. Oncotarget 6: 6931-6943, 2015.

12. Livak KJ and Schmittgen TD: Analysis of relative gene expression data using real-time quantitative PCR and the 2(-Delta Delta C(T)) Method. Methods 25: 402-408, 2001.

13. Huang S, Yuan S, Dong M, Su J, Yu C, Shen Y, Xie X, Yu Y, $\mathrm{Yu} X$, Chen S, et al: The phylogenetic analysis of tetraspanins projects the evolution of cell-cell interactions from unicellular to multicellular organisms. Genomics 86: 674-684, 2005

14. Sala-Valdés M, Ailane N, Greco C, Rubinstein E and Boucheix C: Targeting tetraspanins in cancer. Expert Opin Ther Targets 16: 985-997, 2012

15. Wollscheid V, Kühne-Heid R, Stein I, Jansen L, Köllner S, Schneider A and Dürst M: Identification of a new proliferation-associated protein NET-1/C4.8 characteristic for a subset of high-grade cervical intraepithelial neoplasia and cervical carcinomas. Int J Cancer 99: 771-775, 2002.

16. Brabender J, Marjoram P, Salonga D, Metzger R, Schneider PM, Park JM, Schneider S, Hölscher AH, Yin J, Meltzer SJ, et al: A multigene expression panel for the molecular diagnosis of Barrett's esophagus and Barrett's adenocarcinoma of the esophagus. Oncogene 23: 4780-4788, 2004.

17. Zhang J, Fei B, Wang Q, Song M, Yin Y, Zhang B, Ni S, Guo W, Bian Z, Quan C, et al: MicroRNA-638 inhibits cell proliferation, invasion and regulates cell cycle by targeting tetraspanin 1 in human colorectal carcinoma. Oncotarget 5: 12083-12096, 2014.

18. Chen L, Wang Z, Zhan X, Li DC, Zhu YY and Zhu J: Association of NET-1 gene expression with human hepatocellular carcinoma. Int J Surg Pathol 15: 346-353, 2007.

19. Scholz CJ, Kurzeder C, Koretz K, Windisch J, Kreienberg R, Sauer G and Deissler H: Tspan-1 is a tetraspanin preferentially expressed by mucinous and endometrioid subtypes of human ovarian carcinomas. Cancer Lett 275: 198-203, 2009.

20. Chen L, Zhu Y, Li H, Wang GL, Wu YY, Lu YX, Qin J, Tuo J, Wang JL and Zhu J: Knockdown of TSPAN1 by RNA silencing and antisense technique inhibits proliferation and infiltration of human skin squamous carcinoma cells. Tumori 96: 289-295, 2010.

21. Lu Z, Luo T, Nie M, Pang T, Zhang X, Shen X, Ma L, Bi J, Wei G, Fang $\mathrm{G}$ and Xue X: TSPAN1 functions as an oncogene in gastric cancer and is downregulated by miR-573. FEBS Lett 589: 1988-1994, 2015 